Marina de Tommaso

Vittorio Sciruicchio

Francomichele Puca

\section{Clinical neurophysiology of chronic daily headache}

M. de Tommaso $(\varangle) \cdot$ V. Sciruicchio

F. Puca

Interuniversity Center for the Study of

Headache and Neurotransmitter Disorders,

Neurological Clinic I

University of Bari, Piazza Giulio Cesare 11,

I-70124 Bari, Italy

e-mail: ipnobari@ cimedoc.uniba.it

Tel.: +39-080-5478565

Fax: +39-080-5478532

\begin{abstract}
The pathophysiology of chronic daily headache is unclear. Clinical research aiming to elucidate causative mechanisms is often confounded by the variability of factors involved. Furthermore, there is a recent trend to use neurophysiological tests in the investigation of functional disorders in brain structures. In order to evaluate the pathophysiology of chronic daily headache, several methodologies have been employed. The aim of the present review is to relate the obtained results in the most frequently used neurophysiological tests to possible
\end{abstract}

pathophysiological mechanisms of chronic daily headache. The neurophysiological findings suggest that a common neuronal dysfunction predisposes to primary headaches subtypes, whose chronicization may depend upon many factors, such as the influence of peripheral muscular factors and the dysfunction in central nociceptive modulation. These factors may explain the large variability observed in clinical features.

Key words Chronic daily headache • Neurophysiology $\cdot$ Evoked potential • Electromyography

\section{Introduction}

Chronic daily headache ( $\mathrm{CDH})$ occurs in almost $2 \%$ of general population. Although $\mathrm{CDH}$ is the most frequent headache disorder observed in headache centers [1], it is not clearly classified by International Headache Society (IHS) diagnostic criteria [2]. The clinical variability characterizing $\mathrm{CDH}$ suggests that the underlying causes are heterogeneous.

The pathophysiology of chronic daily headache is still unclear. Clinical research of basic mechanisms is often inconclusive for the variability of the involved factors. Furthermore, there is a recent trend to use neurophysiological tests in the investigation of functional disorders in brain structures. These techniques permit the selective investigation of well-known circuits in the peripheral nervous system, spinal cord and brainstem, although other than expected neurophysiological responses may occur as a consequence of modulation in the complex investigated path- ways. In order to evaluate the pathophysiology of chronic daily headache, several methodologies have been employed.

\section{Reflexology}

Nociceptive flexion reflex

An objective method for measuring pain in humans is represented by the nociceptive flexion reflex (NFR). NFR has two successive components (RII and RIII). The RIII response is associated with pain sensation localized to the stimulation site. Nociceptive flexion reflexes are related to A-delta fiber activation. In normal subjects the value of the reflex threshold is strictly related to that of subjective pain perception.

Sandrini and co-workers [3] observed a reduction of RIII reflex threshold, strictly related to the severity of the 
headache, in patients with migraine with interval headache (chronic almost daily headache).

In 40 patients with chronic tension-type headache (CTTH), Langemark et al. [4] found a high correlation between lower pain tolerance threshold and lower nociceptive flexion reflex threshold. These data confirm the hypothesis that chronic headache represents a disorder of endogenous antinociceptive systems. Indeed, a lower recruitment of descending inhibitory systems probably related to psychological factor may underlie the pathogenesis of CTTH [4].

\section{Exteroceptive suppression of temporalis muscle activity}

Exteroceptive suppression (ES) of muscular activity in the temporalis muscle is an inhibitory brain stem reflex that was applied for the first time by Schoenen in primary headaches [5]. After electrical stimulation of the mental nerve, early (ES1) and late (ES2) exteroceptive silent periods can be recorded over the temples during voluntary jaw occlusion. The second silent period was described to be decreased or abolished in up to $85 \%$ of CTTH sufferers [5], but ES2 abnormalities were unable to distinguish patients with chronic daily headache with drug misuse from those with CTTH, ETTH and migraine. This reflects the heterogeneous pathophysiology of $\mathrm{CDH}$ patients that evolves from tensiontype headache, migraine, or a combination of both. Moreover, various pharmacological agents are able to modulate ES2 duration [6].

Other authors did not confirm these findings, probably for the variability of technical conditions: Gobel et al. [7] failed to detect ES2 abnormalities in a general sample of young chronic tension-type headache sufferers. Similarly, Zwart and Sand found no differences in the duration of ES2 in adult patients with either chronic TTH, migraine, or cervicogenic headache, relative to headache-free controls [8].

In a young adult population, Lipchik et al. [9] examined the ability of pericranial muscle tenderness and the second exteroceptive suppression period to distinguish chronic tension-type headache sufferers, migraine sufferers, and controls. In this study, TTH sufferers exhibited the highest pericranial muscle tenderness, while the shortened ES2 previously observed in adult patients with chronic tension-type headache was not evident in this sample of young headache sufferers. These findings raise the possibility that pericranial muscle tenderness is an early phenomenon in the development of tension-type headache, while ES2 suppression only emerges later in the evolution of the disorder.

In 32 females patients with CTTH, Schoenen et al. [10] observed that temporalis ES2, pericranial electromyography (EMG) level and pain threshold abnormalities did not correlate with each other. These findings suggest that pain is not directly linked to muscle contraction while the reduction or abolition of temporalis second silent period is by far the most frequent pericranial abnormality in CTTH patients. This might be explained by the fact that both nociceptive mechanisms and muscular activity are involved in this electrophysiological test.

On the other hand, Nakashima and coworkers [11, 12] reported a decreased ES2 in idiopathic dystonia as well as in migraine without aura, and postulated that a low level of exteroceptive suppression is unspecific in patients suffering from chronic headaches. The attenuated or absent ES2 response was the major finding in chronic tension-type headache, although it was not confirmed by some researchers, probably for different methodological approaches.

The exact neurophysiological mechanism of the temporalis silent period is not fully understood. It is still unclear whether abnormalities of ES2 in tension-type headache are primary or secondary to headache. The observation of a decreased ES2 duration in chronic pain syndrome without headache [13] suggests that this abnormality is not specific to TTH.

The two silent periods, the early inhibition (ES1) and the late second suppression period (ES2) are supposed to be mediated by separate neural nets in the pontobulbar lateral and medial tegmental fields which receive strong inputs from the periaqueductal gray, amygdala, hypothalamus, orbitofrontal gyrus and limbic structures [14]. These regions play a role in the modulation of nociception, so the duration of the late exteroceptive suppression period could be a marker for the functional state of the pain control system.

Schoenen [15] suggested that a disturbance of limbic pathways to the brainstem (a deficient activation or excessive inhibition) is the common factor underlying the various electrophysiological findings in chronic headache. The influence of neuropsychological factors on the inhibitory interneuronal function may be responsible for the great variability of the silent period duration [16].

\section{Blink reflex}

The blink reflex can give information on peripheral and central trigeminal functions. Electrical stimulation of the supraorbital nerve, in fact, induces a reflex with two different components. The first component (R1), is a short latency response, ipsilateral to the stimulated side and transmitted via a pontine pathway. The second component (R2) is a bilateral, long-latency response mediated via a polysynaptic interneuronal pathway through the lateral reticular formation of the medulla. The R2 component that corresponds to visible closing of lids habituates quickly. A third bilateral 
component of the blink reflex, R3, has been described by Penders and Delwaide [17]. This component has been found to be associated with nociceptive stimuli, to be inhibited by attention and to recover slowly. The relationship between R3 and pain is supported by the observation that R3 is markedly reduced by anesthetic block [18-20].

In migraine, some authors [21] have found an increase in R2 latency, but others [22] have not confirmed this finding. We evaluated the perceptive (Pth) and pain (Path) thresholds and the R1, R2 and R3 thresholds, latencies and amplitudes in 132 adult patients suffering from different forms of headache $[23,24]$. The study population included 61 patients with migraine without aura, 10 patients with migraine with aura, 13 cases of episodic tension-type headache, 16 cases of CTTH, 13 patients with migraine without aura associated with tension-type headache and 19 others with headache that did not fulfill any IHS criteria. In addition, 15 young migraneurs were evaluated. As controls, 28 sex- and age-matched subjects were studied. Pth, Path and R1 and R2 thresholds, latencies and amplitudes were similar in all the studied subjects, whereas R3 distinguished patients from controls. The R3 component was triggered by stimuli of low intensity in headache patients, whereas it appeared only with painful stimuli in controls. Moreover, R3 amplitude was markedly higher in patients than in controls when over Path stimuli were used. The blink reflex R3 component was hyperactive in all patients, regardless of age or type and severity of headache (Figs. 1, 2 ). A dysfunction of the inhibitory pathways on the brainstem circuits may be responsible for the R3 hypersensitivity as well as for the physiopathological mechanisms predisposing to headache.

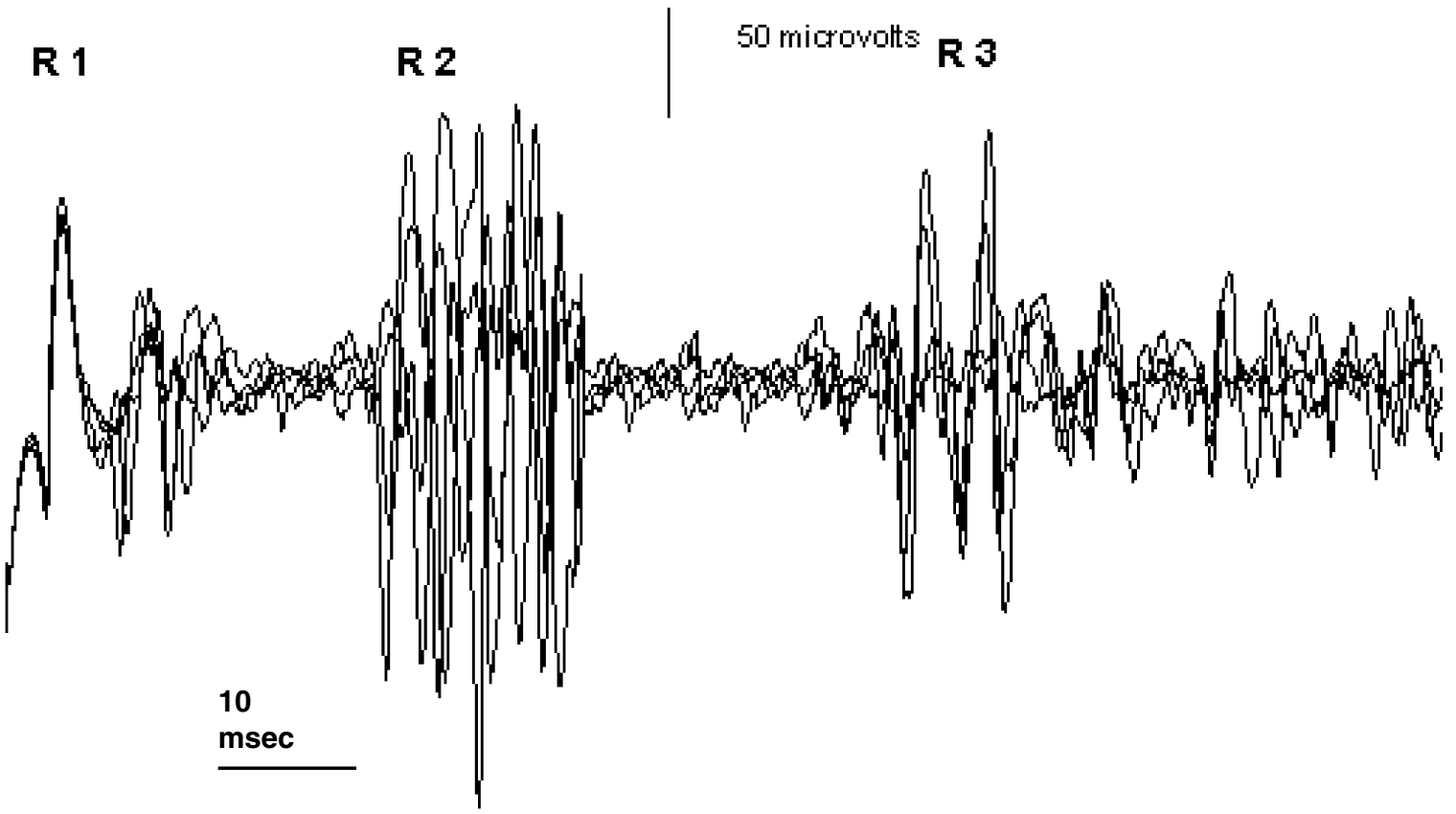

Fig. 1 An example of blink reflex recorded on the right side in a chronic tension-type headache patient. The electrical intensity was $5 x$ perceptive threshold, enough to clearly elicit the R3 component. In this patient, the pain threshold was 12x perceptive threshold 
Fig. 2 Pain (Path), perceptive (Pth) and blink reflex component (R2th, R3th) thresholds in primary headache patients and controls. The R3 threshold reduction is clear in all patients groups. Values are means and standard deviations from ANOVA for unbalanced data. CTTH, chronic tensiontype headache; ETTH, episodic tension-type headache;

$M A$, migraine with aura; $M W O A$, migraine without aura; $T T H$, tensiontype headache; $N C$, not classifiable headache. $* p<0.05 ; * * p<0.01$

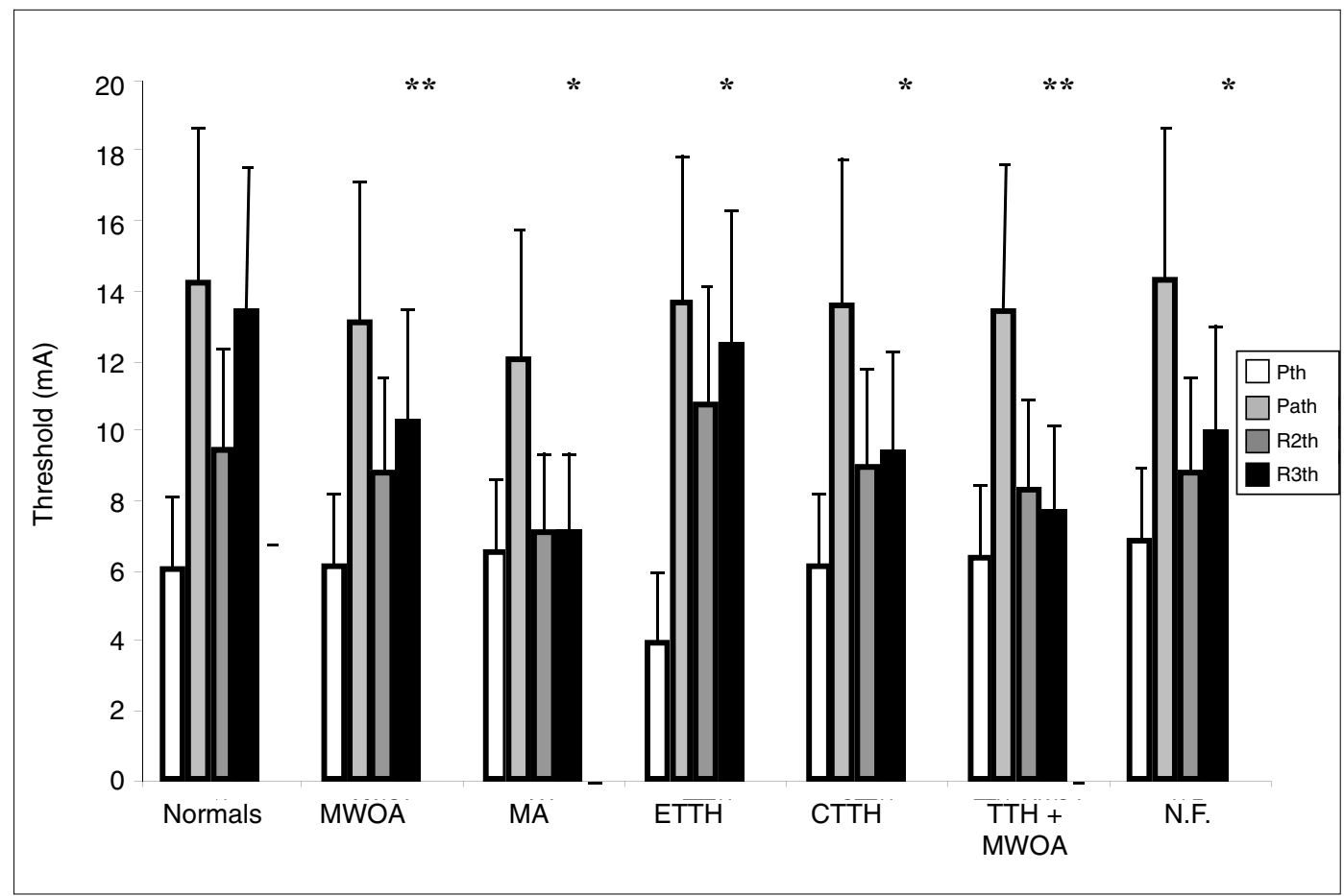

\section{Methodologies for exploring muscular factors}

The importance of pericranial muscles in headache is still unclear. The use of EMG, algometry and manual palpation has been proposed in the IHS classification for the evaluation of pericranial muscle disorders (third digit code) in differentiating patients with TTH or other headaches from healthy subjects.

\section{Pain pressure threshold}

Pain pressure threshold (PPT) is the threshold at which a pressure sensation becomes painful. Pain threshold in pericranial muscles was studied in a random sample of 735 adults by Jensen and Rasmussen [25]. In this study no significant differences were detected in the PPT at the anterior temporal region in patients with CTTH compared to the normal population or to other headache groups. Furthermore, a strong positive correlation between tenderness, evaluated by manual palpation, and frequency of TTH was found, while no relation between tenderness and migraine frequency was seen [26]. Further studies carried out in patients with CTTH reported PPT values in the temporal region not dissimilar from those detected in patients with ETTH or in healthy controls [27].
In other clinical trials, PPT was significantly decreased at frontalis and trapezius muscles in headache patients when compared with controls [28, 29]. Langemark et al. [28] showed that pain thresholds to heat and cold stimulation were significantly decreased in patients with CTTH compared to controls [28]. Furthermore, Schoenen et al. [29] confirmed a lower PPT in the frontalis region as well as over the Achilles tendon in patients with CTTH, in comparison with normal subjects [29]. These conflicting results may be due to different methodologies or to selection bias of headache sufferers: the patients with frequent but not daily headache, examined by Jensen and Rasmussen [25], may be less severely affected than patients with daily occurrence of headache, included in other studies. Although the PPT procedure in CTTH seemed able to relieve a generalized and multimodal increase in pain sensitivity, its usefulness for clinical purposes remains doubtful.

\section{Electromyography}

In order to evaluate the importance of tonic muscle contraction in the pathogenesis of primary headaches, EMG activity in pericranial muscles was assessed in several studies. Jensen et al. [30] performed quantitative EMG evaluation from the right frontal and both temporal muscles in 547 adults. In this study, 15 subjects with CTTH showed higher 
amplitudes in their temporal muscles during rest in comparison with migraineurs, subjects with ETTH and subjects without any experience of headache. In a further study, Jensen [31] observed that EMG levels were unchanged during the critical phase. The decrease in EMG levels during maximal voluntary contraction observed in $\mathrm{CDH}$ patients suggests the importance of peripheral factors such as morphological (relative type II fiber atrophy) and metabolic changes (accumulation of metabolites) in headache chronicization.

\section{Event-related potentials}

Contingent negative variation $(\mathrm{CNV})$ is a negative cerebral potential, first described by Walter et al. [32], recorded over the scalp. CNV is related to attention and arousal, and is modulated by the central catecholaminergic system. The early component of $\mathrm{CNV}$ is dependent on noradrenergic activity (arousal), while the late component is modulated by dopamine (activation).

In a previous study, Schoenen et al. [33] showed an increase in the early component amplitude and a reduced habituation in migraine patients, compared to controls or patients with TTH. On the other hand, Gerber and Schoenen [34] found normal CNV amplitudes and normal habituation in migraineurs during the migraine attack. The normalization of CNV may be due to a depletion of central noradrenergic stores (noradrenergic arousal system) which reduces the threshold for a migraine attack; alternatively it may be due to a reduction of attention during the headache attack [35].

In a recent study, during a 3-s interstimuli interval, amplitude and habituation of total CNV and of post-imperative negative variation (PINV) were calculated in 15 women suffering from migraine without aura, in 15 women diagnosed with $\mathrm{CDH}$ evolved from migraine without aura with interparoxysmal CTTH, and in 15 healthy women [35]. In this study, according to previous observations [33, 36], migraine patients were characterized by a more pronounced negativity of the early component and total CNV, compared to $\mathrm{CDH}$ sufferers and controls. $\mathrm{CDH}$ patients evolved from migraine showed a reduced negativity of the late component and a pronounced PINV compared to migraineurs and healthy subjects, while both patient groups showed reduced habituation of the early CNV component. The former abnormality, observed in major depression and anxiety disorders, may be due to the worsening of disease while the latter fea- ture, independently from the clinical course of disease, may be a basic characteristic of migraine brain [37]. The authors concluded that $\mathrm{CNV}$ features may be predictive of transformed migraine.

\section{Discussion}

The use of neurophysiological procedures in chronic daily headache can contribute to confirm disorders in pericranial pain sensitivity and/or possible abnormalities in central pain modulation. Nevertheless, results obtained by neurophysiological methods suffer from some methodological limitations due to patient selection bias and variability in technical procedures.

The results from studies of blink reflex, evoked potentials and event-related potentials confirm the existence of central dysfunctions predisposing to migraine as well as to TTH. Schoenen hypothesized exclusively a central mechanisms underlying TTH chronicization, on the basis of the decreased exteroceptive silent period in patients with CTTH [5]. The abnormal ES2 responses observed in CTTH may reflect an excessive inhibition of brainstem interneurons secondary to a disturbed limbic control structures such as the periacqueductal gray and raphe magnus nucleus. Moreover, $\mathrm{CNV}$ features may be predictive of transformed migraine.

A purely central hypothesis fails to explain the relation between headache and peripheral factors. Jensen et al. [30] suggested that the chronic accumulation of metabolites due to a persistent involvement of the pericranial muscles explains the EMG findings in patients with CTTH [38, 39]. Indeed, involuntary contraction of muscles, due to a mechanical or psychological stress, causes increased peripheral input from mechanoreceptors and afferent fibers. A peripheral mechanism of TTH can explain episodic subtype, whereas a secondary, segmental central sensitization and/or an impaired supraspinal modulation of incoming stimuli seems to be involved in subjects with CTTH. Prolonged nociceptive stimuli from myofascial tissue may be a fundamental factor in the conversion from episodic to chronic TTH.

Neurophysiological findings suggest that a common neuronal dysfunction can predispose to primary headaches subtypes, whose chronicization may depend upon many factors, such as the influence of peripheral muscles and a dysfunction in central nociceptive modulation. These factors may explain the large variability observed in clinical features. 


\section{References}

1. Mathew NT (1991) Chronic daily headache: clinical features and natural history. In: Nappi G, Bono G, Sandrini G, Martignoni E, Micieli G (eds) Headache and depression. Raven, New York, pp 49-58

2. Headache Classification Committee of the International Headache Society (1988) Classification and diagnostic criteria for headache disorders, cranial neuralgias and facial pain. Cephalalgia 8[Suppl 7]:1-96

3. Sandrini G, Arrigo A, Bono G, Nappi G (1993) The nociceptive flexion reflex as a tool for exploring pain control systems in headache and other pain syndromes. Cephalalgia 13:21-27

4. Langemark M, Bach FW, Jensen TS, Olesen J (1993) Decreased nociceptive flexion reflex threshold in chronic tension-type headache. Arch Neurol 50:1061-1064

5. Schoenen J (1993) Wolff Award 1992. Exteroceptive suppression of temporalis muscle activity in patients with chronic headache and in normal volunteers: methodology, clinical and pathophysiological relevance. Headache 33:3-17

6. Schoenen J, Sianard-Gainko J, Raubuchl O (1992) Serotonin and temporalis exteroceptive silent periods. In: Olesen J, Saxena PR (eds) 5-Hydroxytryptamine mechanisms in primary headaches. Raven, New York, pp 91-97

7. Gobel H, Ernst M, Jeschke J, Keil R, Weigle L (1992) Acetylsalicylic acid activates antinociceptive brain stem reflex activity in headache patients and in healthy subjects. Pain 48:187-196

8. Zwart JA, Sand T (1995) Exteroceptive suppression of temporalis muscle activity: a blind study of tension-type headache, migraine and cervicogenic headache. Headache 35:338-343

9. Lipchik GL, Holroyd KA, France CR, Kvaal SA, Segal D, Cordingley GE, Rokicki LA, McCoc HR (1996) Central and peripheral mechanisms in chronic tension-type headache. Pain 64:467-475
10. Schoenen J, Gerard P, De Pasqua V, Sianard-Gainko J (1991) Multiple clinical and paraclinical analyses of chronic tension-type headache associated or unassociated with disorder of pericranial muscles. Cephalalgia 11:135-139

11. Nakashima K, Takahashi K (1991) Exteroceptive suppression of the masseter, temporalis and trapezius muscles produced by mental nerve stimulation in patients with chronic headaches. Cephalalgia 11:23-28

12. Nakashima K, Thompson PD, Rothwell JC, Day BL, Stell R, Marsden CD (1989) An exteroceptive reflex in the sternocleidomastoid muscle produced by electrical stimulation of the supraorbital nerve in normal subjects and patients with spasmodic torticollis. Neurology 39:1354-1358

13. Wallasch T, Lindner V, Soyka D (1992) Temporalis-inhibitory reflex in the assessment of the functional state of the pain control system in chronic headache sufferers. Headache Quarterly 3:431-433

14. Cruccu G, Bowsher D (1986) Intracranial stimulation of the trigeminal nerve in man II. Reflex responses. J Neurol Neurosurg Psychiatry 49:419-427

15. Schoenen J (1990) Tension-type headache: pathophysiologic evidence for a disturbance of "limbic" pathways of the brain stem. Headache 30:314-315

16. Paulus W, Raubuchl O, Straube A, Schoenen J (1992) Exteroceptive suppression of temporalis muscle activity in various types of headache. Headache 32:41-44

17. Penders CA, Delwaide PJ (1973) Physiological approach to the human blink reflex. In: Desmedt JE (ed) New developments in electromyography and clinical neurophysiology, vol. 3. Karger, Basel, pp 649-657

18. Rossi B, Risaliti R, Rossi A (1989) The R3 component of the blink reflex response induced by activation of high threshold cutaneous afferents. Electroencephalogr Clin Neurophysiol 73:334-340
19. Rossi B, Vignocchi G, Mazzoni M, Pardossi L, Bianchi F, Muratorio A (1993) Causes of the instability of R3 component of electrically evoked blink reflex: role of the attention to the stimulus. Electromyogr Clin Neurophysiol 33(1):49-53

20. Ellrich J, Broom B, Hopf HC (1997) Pain-evoked blink reflex. Muscle Nerve 20(3):265-267

21. Bank J, Bense E, Kiralj C (1992) The blink reflex in migraine. Cephalalgia 12(5):289-292

22. Sand T, Zwart JA (1994) The blink reflex in chronic tension type headache, migraine, and cervicogenic headache. Cephalalgia 14(6):447-450

23. De Tommaso M, Sciruicchio V, Guido M, Sasanelli G, Tota P, Puca FM (1997) Pain sensitivity in migraine and tension type headache: an electrophysiological evaluation by the R3 . Cephalalgia 17(3):P356

24. de Tommaso M, Guido M, Libro G, Sciruicchio V, Puca FM (2000) The three responses of the blink reflex in adult and juvenile migraine. Acta Neurologica Belg 100:96

25. Jensen R, Rasmussen BK (1996) Muscular disorders in tension-type headache. Cephalalgia 16:97-103

26. Jensen R, Olesen J (1996) Initiating mechanisms of experimentally induced tension-type headache. Cephalalgia 16:175-182

27. Schoenen J, Bottin D, Hardy F, Gerard P (1991) Cephalic and extracephalic pressure pain thresholds in chronic tension-type headache. Pain 47:145-149

28. Langemark M, Jensen K, Jensen TS, Olesen J (1989) Pressure pain thresholds and thermal nociceptive thresholds in chronic tension-type headache. Pain 38:203-210

29. Schoenen J, Gerard P, De Pasqua V, Sianard-Gainko J (1991) Multiple clinical and paraclinical analyses of chronic tension-type headache associated or unassociated with disorder of pericranial muscles. Cephalalgia 11:135-139

30. Jensen R, Fuglsang-Frederiksen A, Olesen J (1994) Quantitative surface EMG of pericranial muscles in headache. A population study. Electroencephalogr Clin Neurophysiol 93:335-344 
31. Jensen R (1995) Mechanisms of spontaneous tension type headaches: an analysis of tenderness, pain thresholds and EMG. Pain 64:251-256

32. Walter WG, Cooper R, Aidridge VJ, McCallum WC, Winter AL (1964) Contingent negative variation: an electric sign of sensorimotor association and expectancy on the human brain. Nature 203:380-384

33. Schoenen J, Maertens A, TimsitBerthier M, Timsit M (1985) Contingent negative variation (CNV) as a diagnostic and physiopathological tool in headache patients. In: Clifford Rose FL (ed) Migraine. Proceedings of the Fifth International Migraine Symposium, London 1984 Karger, Basel, pp 17-25
34. Gerber WD, Schoenen J (1998) Biobehavioral correlates in migraine: the role of hypersensitivity and information-processing dysfunction. Cephalalgia 18[Suppl 21]:5-11

35. Siniatchkin M, Gerber W-D, Kropp P, Vein A (1998) Contingent negative variation in patients with chronic daily headache. Cephalalgia 18:565-569

36. Bocker KBE, Timsit-Berthier M, Schoenen J, Brunia CHM (1990) Contingent negative variation in migraine. Headache 30:604-609
37. Kropp P, Gerber WD (1993) Is increased amplitude of contingent negative variation in migraine due to cortical hyperactivity or to reduced habituation? Cephalalgia 13:37-41

38. Jensen R, Rasmussen BK, Pedersen B, Olesen J (1993) Muscle tenderness and pressure pain thresholds in headache. A population study. Pain 52:193-199

39. Jensen R (1999) Pathophysiological mechanisms of tension type headache: a review of epidemiological and experimental studies. Cephalalgia 19:602-621 